DOI: http://dx.doi.org/10.18309/anp.v1i48.1253

\title{
UM NOVO OLHAR SOBRE AS SENTENÇAS COM REDOBRO EM PORTUGUÊS BRASILEIRO
}

\section{A NEW VIEW ON DOUBLE SUBJECT SENTENCES IN BRAZILIAN PORTUGUESE}

Sandra Quarezemin Universidade Federal de Santa Catarina, Florianópolis, Santa Catarina, Brasil

Resumo: O objetivo principal desta pesquisa é investigar o comportamento do sujeito e do pronome resumptivo nas sentenças com redobro do sujeito em português brasileiro (PB). A partir de evidências sintáticas, semânticas e prosódicas, será possível verificar (i) se tais construções podem ser analisadas como casos de deslocamento à esquerda; (ii) se essas sentenças caracterizam o PB como uma língua tópico proeminente; (iii) se o sujeito pré-verbal se comporta como um tópico; (iv) se o sujeito tem o mesmo comportamento do objeto deslocado com retomada pronominal; (v) se o pronome resumptivo que retoma o sujeito préverbal nessas construções deve ser analisado como um clítico (um núcleo) ou como um pronome fraco, ocupando a posição de especificador. A hipótese deste trabalho é a de que o pronome resumptivo pode instanciar visivelmente o núcleo da projeção funcional SubjP (RIZZI, 2005), sendo a realização lexical de Subj, atrai um constituinte compatível com o traço "sujeito da predicação" (cf. CARDINALETTI, 1997, 2004).

Palavras-chave: Duplicação do Sujeito; Pronome Resumptivo; Cartografia; Português Brasileiro

Abstract: The main objective of this research is to investigate the behavior of the subject and the resumptive pronoun in the double subject constructions in Brazilian Portuguese (BP). From syntactic, semantic and prosodic evidences, it will be possible to verify (i) if such constructions can be analyzed as cases of left dislocation; (ii) if these sentences characterize $\mathrm{BP}$ as a prominent topic language; (iii) if the pre-verbal subject behaves as a topic; (iv) if the subject has the same behavior of the dislocated object with the resumption of the pronoun; (v) if the resumptive pronoun that recovers the pre-verbal subject in these constructions should be analyzed as a clitic (a head) or as a weak pronoun, occupying the position of specifier. The hypothesis of this work is that the resumptive pronoun can visibly instantiate the head of the SubjP functional projection (RIZZI, 2005), being the lexical realization of Subj, attracts a constituent compatible with the feature "subject of predication" (cf. CARDINALETTI, 1997, 2004).

Keywords: Double Subject; Resumptive Pronoun; Cartography; Brazilian Portuguese 


\section{INTRODUÇÃO}

Este trabalho consiste em um estudo sintático sobre o comportamento do sujeito e do pronome resumptivo nas sentenças com redobro no Português Brasileiro (doravante PB). A pesquisa sobre a sintaxe do sujeito deve ler em conta certas propriedades formais deste constituinte: (i) ser identificado de forma "única": toda sentença tem um sujeito; (ii) ser obrigatório: línguas não 'pro-drop' exibem sujeito expletivo onde não há um sujeito lexical; (iii) concordar com o verbo finito; (iv) ser hierarquicamente mais alto na estrutura; (v) a sua extração é mais restrita do que a de outros constituintes; (vi) pode ser nulo em sentenças imperativas e infinitivas; (vii) ser normalmente relacionado ao tópico (QUAREZEMIN, 2017a).

Quarezemin (2017a; 2018) e Quarezemin e Cardinaletti (2017) mostram que o sujeito pré-verbal, em PB, tem um comportamento distinto do tópico. Essa distinção já foi mostrada em italiano (CARDINALETTI, 1997, 2004; RIZZI, 2005), em espanhol (SUÑER, 2003) e em português europeu (COSTA; DUARTE, 2002; COSTA, 2010), todas línguas de proeminência de sujeito, fato que nos leva a questionar a classificação do PB como uma língua de proeminência de tópico.

Este estudo parte da hipótese de que o PB apresenta dois tipos de estruturas com redobro do sujeito pré-verbal: uma com pronome fraco e uma com pronome forte (QUAREZEMIN, 2018). Apenas no último caso é que o sujeito está deslocado na periferia esquerda da sentença, como um tópico. Quando a duplicação do sujeito ocorre com o pronome fraco, o sujeito está na posição mais alta do domínio TP, em Spec,SubjP (cf. CARDINALETTI, 2004; RIZZI, 2006; RIZZI; SHLONSKY, 2006; 2007). O redobro do sujeito não deve ser sempre identificado como uma estratégia de topicalização deste constituinte.

\section{A SINTAXE DO SUJEITO NO PORTUGUÊS BRASILEIRO}

Para alguns autores, o sujeito pré-verbal no PB está deslocado à esquerda, em uma posição no domínio CP (cf. GALVES, 2001; KATO; NEGRÃO, 2000), indo ao encontro do que tem sido afirmado para as línguas de sujeito nulo (ALEXIADOU; ANAGNOSTOPOULOU, 1998; BARBOSA, 2000, 2006; ORDÓÑEZ, 1997). Outros autores, contudo, defendem que em línguas de sujeito nulo o sujeito pré-verbal ocupa uma posição no domínio TP (cf. CARDINALETTI, 1997; COSTA, 2001; COSTA; DUARTE, 2002; GOODALL, 1999, 2001; SUÑER, 2003).

O sujeito no PB tende a ocupar a posição pré-verbal tanto na sentença interrogativa quanto na declarativa, como em (1), até mesmo em contextos nos quais o sujeito aparece focalizado, como em (2). Já no italiano, a posição estrutural do sujeito está condicionada a, pelo menos, dois fatores: (i) classe do verbo e (ii) status discursivo do sujeito, como em (3) e $(4)^{1}$.

(1) a. O que João fez? João quebrou o vaso.

b. O que João fez ontem? João dançou.

c. Que horas João chegou ontem? João chegou às 9 h.

(2) a. Quem quebrou o vaso? João quebrou o vaso.

b. Quem espirrou? João espirrou.

c. Quem chegou? João chegou.

\footnotetext{
${ }^{1}$ As sentenças em (3) e (4) são as traduções em italiano das sentenças em (1) e (2), respectivamente.
} 
(3) a. Cosa ha fatto Gianni? Gianni ha rotto il vaso.

b. Cosa ha fatto Gianni ieri sera? Gianni ha ballato.

c. Che ora è arrivato Gianni ieri sera? Gianni è arrivato alle 9.

(4) a. Chi ha rotto il vaso? L'ha rotto Gianni.

b. Chi ha starnutito? Ha starnutito Gianni.

c. Chi è arrivato? E' arrivato Gianni.

As sentenças em (1) e (2) nos mostram que independentemente de o sujeito ser uma informação compartilhada (1) ou não (2) a posição pré-verbal é escolhida; o tipo do verbo (transitivo, intransitivo, inacusativo) também não interfere no posicionamento do sujeito.

Quando observamos alguns dados do italiano vêneto (cf. CARDINALETTI, 2012), percebemos uma certa proximidade com uma língua de sujeito nulo parcial como o PB.

(5) a. Chi ha rotto il vaso? Gianni l'ha rotto.

b. Chi ha starnutito? Gianni ha starnutito.

(5') a. GIANNI l'ha rotto, non Maria

b. GIANNI ha starnutito, non Maria

Em (5), o sujeito Gianni está focalizado e, ainda assim, ocupa a posição pré-verbal. Somente com verbos inacusativos, como em (5c), é que o DP pode ser posposto. Em (5'), o sujeito Gianni é foco corretivo (cf. RIZZI, 2018). Apenas com verbos inacusativos é que o sujeito aparece posposto, como em Chi è arrivato? È arrivato Gianni. Cardinaletti (2012) propõe que neste caso o DP está na mesma posição do objeto, conforme a hipótese inacusativa de Burzio (1986). Segundo a autora, os falantes do italiano vêneto apresentam um padrão opcional (sujeito pós-verbal como o italiano e sujeito pré-verbal como os dialetos do norte) típico de situação de contato linguístico. Cardinaletti (2012) afirma que os dialetos do norte da Itália (DNI) não são línguas pro-drop, são similares às línguas pro-drop parcial, como o finlandês, o hebraico e o PB. Os DNI, como os dados do fiorentino e de Conegliano abaixo, exibem pronomes expletivos, como em (6), e o sujeito pós-verbal é mais restrito do que em italiano, como em (7).

(6) a. Gli è venuto la Maria. $\quad$ (Fiorentino) expl chegou a Maria

b. Gli ha telefonato delle ragazze expl telefonou algumas garotas

(BRANDI; CORDIN, 1981; 1989)

(7) a. El è riva la Maria. expl chegou a Maria

(Conegliano, Vêneto)

b. ${ }^{*}$ El a ridest la Maria, al cinema. expl riu a Maria, no filme

c. *Me ha scrit un poema la Maria. clit-pra mim escreveu um poema a Maria

(SACCON, 1993)

Além de o PB reservar preferencialmente a posição pré-verbal para o sujeito, independente de fatores semântico-discursivos (cf. QUAREZEMIN, 2005; 2009), também permite que ocorra uma duplicação do sujeito por meio de um pronome, como em (9). 
(9) a. A Clarinhai ela cozinha que é uma maravilha.

b. Eu acho que um trabalho sério ele $_{i}$ teria que começar por aí.

c. Vocêi, no Canadá, você $\hat{i}_{i}$ pode ser o que quiser.

(DUARTE, 2000, p. 28)

Os dados em (9) nos mostram que o redobro pode ocorrer na sentença matriz (9a), na encaixada (9b) e com material interveniente entre o sujeito e o pronome resumptivo (9c). Logo podemos questionar: em todas essas sentenças o sujeito e o pronome resumptivo têm o mesmo comportamento?; todas elas devem ser analisadas como casos de deslocamento à esquerda do sujeito?; nessas sentenças, o sujeito pré-verbal se comporta como um tópico?. É necessário comparar as sentenças com redobro do sujeito sem e com material interveniente. A hipótese é que temos dois tipos de construção com redobro do sujeito no PB: (i) sentenças com deslocamento à esquerda e sujeito topicalizado, de um lado, e (ii) sentenças com o que vamos chamar de redobro simples, estando o sujeito em uma posição argumental, não deslocada. Apenas quando há um material interveniente, ou uma pausa marcada, entre o sujeito e o pronome temos uma situação real de deslocamento à esquerda.

(10) A empresa $a_{i}$, eles $_{i}$ reembolsaram passagem, hospedagem, alimentação...

(11) Espírito $_{i} \ldots$ eles $_{\mathrm{i}}$ ficam preso aqui na terra.

(12) $\mathrm{O} \mathrm{EPA}_{i}$, hoje em dia eles $s_{i}$ têm a preferência de mesclar.

(SOUZA, 2007, p. 111).

Nas sentenças acima, o DP sujeito é singular e retomado por um pronome plural. Parece que o pronome tem uma interpretação arbitrária, acionando uma leitura genérica, não referencial. A concordância não é realizada com o DP sujeito, mas sim com o pronome ${ }^{2}$. Nossa hipótese é que o pronome das sentenças em (10-12) não é do mesmo tipo que o pronome das sentenças em $(9 a, b)$, estando cada um deles em posições estruturais distintas. Em (10-12), o pronome é forte e ocupa uma posição de especificador, enquanto em (10a,b), o pronome é realizado como um núcleo. pronome clítico:

Nos DNI, contrário ao francês, também é possível a retomada do sujeito pelo

(13) a. Nessuno gl'ha detto nulla. (fiorentino)

a' Nisun l'ha dit niente. (trentino) ninguém ele disse nada

(14) b. Tutto gl'è sucesso di notte. (fiorentino)

b' Tut l'è capita de not. (trentino) tudo ele aconteceu de noite

(SILVA, 2006, p. 193-194)

No PB, diferente do que ocorre acima, verificamos que a retomada de um

${ }^{2}$ Há casos em que o pronome parece funcionar como um expletivo (cf. VITRAL; RAMOS, 2006):

(i) Na universidade você vê de tudo.

Este uso também pode estar relacionado a uma nova estratégia de indeterminação do sujeito vem aparecendo: o uso de pronomes e expressões nominais no lugar do uso do pronome se ou do verbo na terceira pessoa plural (cf. LUNGUINHO; MEDEIROS JÚNIOR, 2006; FUCHSBERGER, 2018)

(ii) a. O cara viaja e sempre esquece alguma coisa.

b. Tu encontra de tudo no mercado público. 
quantificador universal nu (bare), sem uma restrição lexical, não é possível. A natureza do elemento pronominal pode estar por trás dessa distinção. Os DNI têm um sistema de clítico sujeito, enquanto o PB não. Ainda assim, parece possível associar o pronome das construções de redobro simples do $\mathrm{PB}$ a um traço de pessoa.

\section{AS SENTENÇAS COM REDOBRO DO SUJEITO}

Duarte e Soares da Silva (2016) apontam que o PB vem passando por um processo de mudança sintática que, além de afetar referentes [-humanos], exibe uma expressiva ocorrência de sujeitos deslocados à esquerda. Os autores verificam que tais estruturas (i) podem aparecer na sentença matriz ou encaixada, (ii) o elemento deslocado pode estar adjacente ou não ao pronome correferente, (iii) não são restritas pelos traços semânticos do referente.

(15) a. Eu acho que [o povo brasileiro] ele $_{i}$ tem uma grave doença.

b. [O comércio] $]_{i}$ eu acho que ele $e_{i}$ é sábio ${ }^{3}$.

c. Em qualquer dessas cidades, [um homem comum] $]_{i}$ ele $e_{i}$ tem um conforto compatível com a dignidade de uma pessoa humana, entendeu?

d. [Um apartamento com um banheiro só $]_{i} e e_{i}$ já vale menos.

(DUARTE; SOARES DA SILVA, 2016, p. 19-20)

Nas sentenças acima, temos a ocorrência de DPs distintos [+hum/+spec], [hum/+spec], [+hum/-spec] e [-hum/-spec]. Duarte e Soares da Silva (2016) apontam que das 52 ocorrências de deslocamento à esquerda encontradas na amostra, 24 sentenças apresentam o DP [+hum/+spec], 21 com DP [-hum/+spec], 4 com DP [+hum/-spec], e 3 sentenças com DP [-hum/-spec]. Partindo desses números, observamos uma preferência pelo DP [+spec]. Das 52 ocorrências, apenas 7 sentenças tinham o DP [-spec], o que parece sinalizar que o traço de especificidade, de alguma forma, restringe a ocorrência deste tipo de estrutura.

Para Duarte (2000), as sentenças com redobro do sujeito sinalizam que o PB está se tornando uma língua não pro-drop, como o francês e o inglês. A autora também relaciona o aparecimento dessas construções ao enfraquecimento da morfologia de flexão verbal e à perda do Princípio Evite Pronome no PB. Segundo Kato (1999, também assumido em Barbosa, Duarte e Kato (2005)), EPP não pode mais ser satisfeito pelo afixo de concordância no PB, disparando, então, o movimento do sujeito para Spec,IP e criando um paradigma de pronomes nominativos fracos.

Análises que assumem o redobro do sujeito pré-verbal como um caso de deslocamento à esquerda (do tipo que ocorre em francês) não diferenciam as estruturas que têm um pronome resumptivo forte das construções que apresentam uma retomada por um pronome fraco, como em muitas das sentenças com redobro do sujeito pré-verbal no PB. Também não diferenciam as estruturas com objeto tópico deslocado das sentenças com sujeito duplicado, já que em ambos os casos tanto o objeto quanto o sujeito estarão em uma posição na periferia esquerda da sentença (no domínio $\mathrm{CP}$ ) e o pronome em posição argumental.

A hipótese deste artigo é que o PB apresenta dois tipos de estruturas com redobro do sujeito pré-verbal: uma com pronome fraco e uma com pronome forte. Apenas no último caso é que o sujeito está deslocado na periferia esquerda da sentença. Quando a duplicação do sujeito ocorre com pronome fraco, o sujeito está na posição mais alta do domínio TP, em Spec,SubjP. O redobro do sujeito não deve ser identificado como um caso de deslocamento

\footnotetext{
${ }^{3}$ Apenas nesta sentença é que temos um caso explícito de topicalização com deslocamento à esquerda do sujeito da encaixada.
} 
deste constituinte ou como uma estratégia de topicalização, já que a duplicação ocorre até mesmo onde um tópico não é permitido.

Camacho $(2013 ; 2016)$ afirma que as sentenças com redobro do sujeito, que o autor denomina de subject clitic left deslocation (SCLLD), não têm as restrições sintáticas e semânticas que restringem as sentenças com objeto deslocado à esquerda e retomado pelo clítico em outras variedades românicas, uma vez que as sentenças com duplicação do sujeito podem ocorrer na matriz ou encaixada, ter interpretação arbitrária, o DP pode ser definido ou indefinido, entre outras propriedades. A nossa hipótese é que isso ocorre justamente porque em muitas das sentenças com redobro do sujeito não há nenhum tipo de deslocamento, como ocorre com as sentenças com objeto clitic left deslocation (um caso típico de topicalização na periferia esquerda da sentença). Assumindo uma análise cartográfica, defendemos que o sujeito figura em uma posição argumental, a mais alta do domínio flexional, mantendo a estrutura informacional sujeito-predicado.

Silva (2006, p. 195-196), contra o redobro como estrutura de deslocamento à esquerda do sujeito, observa que a ausência de uma pausa entre o DP sujeito e o pronome fraco não causa agramaticalidade:

(16) a. A Clarinha $a_{i}$ ela $a_{i}$ cozinha que é uma maravilha.

b. Eu acho que o povo brasileiro ele $_{i}$ tem uma grave doença.

c. Eu acho que um trabalho sério ele $_{i}$ teria que começar por aí.

(DUARTE, 2000, p. 28)

Além disso, a quebra da adjacência entre o DP sujeito e o pronome fraco, por meio de XPs, compromete a gramaticalidade da sentença:

(17) a. *??Você intencionalmente/ sempre cê lê livros românticos.

b. ??Você, sua mulher, cê deve tratar ela bem.

(SILVA, 2004, p. 439)

Silva (2006) ainda verifica que a duplicação de sujeitos quantificados por "todo(s)" e "cada" não torna a frase agramatical:

(18) a. Todo homem ele odeia se sentir fraco.

b. Cada menino ele leva um livro para a escola.

Quarezemin (2018) acrescenta a possibilidade da extração como mais uma propriedade sintática contra o redobro como um caso de sujeito tópico:

(19) a. Onde Pedro acha que a Ana ela encontrou João?

b. *Onde Pedro acha que João a Ana (ela) encontrou?

Além dessas propriedades, Silva (2006, p. 196), destaca o efeito de ligação-A (que pode ser atestado nas sentenças com redobro) como evidência de que o sujeito e o pronome resumptivo ocupam uma posição argumental.

(20) As mulheres ${ }_{i}$ elas ${ }_{i}$ usaram todas os brincos umas das outras ${ }_{i}$.

Se nem todas as construções com redobro correspondem à retomada de um elemento deslocado, e estando o sujeito pré-verbal e o pronome fraco em uma posição-A alta na sentença, sem quebra de adjacência, qual é a posição do DP sujeito e do pronome resumptivo? 
Qual é o estatuto do pronome? O pronome resumptivo nas construções de redobro do sujeito do PB pode ser analisado como a realização lexical de um núcleo que porta um traço de Pessoa?

Rizzi (2015) discute duas hipóteses para o movimento do sujeito da posição temática para o Spec de um núcleo flexional: (i) hipótese padrão - movimento é motivado por requerimentos de Caso, relevância da interface com a morfologia; (ii) hipótese alternativa - a posição sujeito é dotada de propriedades discursivas (quase-topicalidade) e sistemas externos requerem que eventos sejam expressos na forma sujeito-predicado (ROTHSTEIN, 1983), relevância da interface com a semântica (que motiva o movimento).

Segundo Rizzi (2015; 2018), as línguas que têm um sistema de clíticos separado da morfologia do verbo flexionado, como é o caso da maioria dos dialetos do norte da Itália (cf. POLETTO, 2000; MANZINI; SAVOIA, 2005), podem instanciar visivelmente o núcleo Subj.

(21) El fio el mangia l pom. (milanese) o menino Subj come a maçã

Quanto a EPP e as posições de sujeito, Rizzi (2015) observa que se a posição canônica do sujeito não é Spec,TP, mas sim Spec,SubjP, para onde o sujeito se move para satisfazer o Critério Sujeito, o traço EPP de T torna-se redundante. De acordo com a abordagem criterial, EPP é uma propriedade apenas dos núcleos criteriais, construída na satisfação da configuração criterial. Se T não é um núcleo criterial, segue que a atração de um nominal para o seu especificador pode servir apenas para facilitar o movimento para Spec,SubjP, a posição criterial. Mas de que forma a passagem por Spec,TP facilita a satisfação do Critério Sujeito?

Rizzi (2014) assume que número é um traço-phi de T. Ambos T e Subj têm um traço $\mathrm{D}$ (eles sondam uma categoria nominal), mas os atributos de $\mathrm{D}$ são diferentes: $\mathrm{D}$ em $\mathrm{T}$ é [número], enquanto D em Subj é [pessoa]:

\section{(22) Subj (D [pessoa]) T (D [número])}

Subj além de ser um núcleo criterial, participa do sistema phi-Case, à medida que sonda um D [pessoa]. Assumindo que o traço D em T atua como um interventor, bloqueará a sonda pelo Subj. Para evitar este efeito de intervenção, o sujeito deve ser movido para uma posição fora do domínio de c-comando de T. O movimento através de Spec,TP é, então, forçado. Uma derivação na qual $\mathrm{T}$ concorda com o sujeito sem atrai-lo e o sujeito se move diretamente para Spec,SubjP não é permitida segundo essa análise ${ }^{4}$.

$$
\begin{aligned}
& \text { (23) a. [SubjP DP }\left[\text { [subj ele }\left[\mathrm{TP}_{\mathrm{i}}[\mathrm{T}, \mathrm{V}(\ldots)]\right]\right. \\
& \text { b. [TорP DP [SubjP ele } e_{i}\left[\operatorname{Subj}_{1}\left[\mathrm{TP}_{\mathrm{i}}[\mathrm{T}, \mathrm{V}(\ldots)]\right]\right.
\end{aligned}
$$

Em (23a), temos uma estrutura compatível com as sentenças que estamos chamando aqui de simples redobro, sem deslocamento. Quarezemin (2018) propõe que, neste caso, o pronome resumptivo é a realização de Subj, como ocorre com o clítico sujeito nos DNI (cf. RIZZI, 2015; 2018), e o DP sujeito ocupa a posição Spec,SubjP, tendo a estrutura informacional sujeito-predicado. Já em (23b), a estrutura resgata as sentenças com

\footnotetext{
${ }^{4}$ Rizzi (2015) observa que o sujeito deve ser capaz de contornar TP e se mover diretamente para Spec,SubjP quando ele não é sondado por quaisquer traços-phi de T. Por exemplo, quando o sujeito não depende dos traços de T para Caso, como na ocorrência do sujeito pré-verbal PP locativo.
} 
deslocamento à esquerda do sujeito e retomada pronominal. Observamos que, neste caso, o DP sujeito terá as propriedades do tópico e o pronome resumptivo forte ocupa Spec,SubjP, tendo a estrutura informacional tópico-comentário.

\section{CONSIDERAÇÕES FINAIS}

Este estudo mostrou que nem todas as sentenças com sujeito duplicado do PB devem ser analisadas como estruturas de deslocamento à esquerda. Em muitas delas, o sujeito não se comporta como um tópico nas sentenças com redobro. Além disso, tais sentenças não servem como evidência de que o PB é uma língua de proeminência de tópico, tendo a estrutura default tópico-comentário.

O pronome resumptivo nas sentenças com o que denominamos de redobro simples do PB tem o comportamento de um clítico sujeito, é a realização lexical de Subj, atraindo um nominal para Spec,SubjP. O núcleo Subj tem propriedades híbridas: incorpora um critério, pertencendo à classe que inclui WH, FOC e Rel, e também participa do sistema phi-Case. $\mathrm{O}$ movimento do sujeito para Spec,TP não é regido pelo traço EPP. Segundo Rizzi (2015), T intervém na sondagem de Subj, por isso o sujeito nominal deve figurar acima desse núcleo, a fim de estar em uma posição acessível para ser sondado por Subj.

\section{Referências}

ALEXIADOU, Artemis; ANAGNOSTOPOULOU, Elena. Parametrizing AGR: Word Order, V-Movement and EPP-Checking, Natural Language and Linguistic Theory, 16(3): 491-531, 1998.

BARBOSA, Pilar. Clitics: A window into the null subject property. In: COSTA, J. (ed.), Portuguese syntax: New comparative studies. Oxford: Oxford University Press, 2000.

BARBOSA, Pilar. Ainda a questão dos sujeitos pré-verbais em PE: Uma resposta a Costa (2001). D.E.L.T.A. 22.2: 345-402, 2006.

BARBOSA, Pilar; DUARTE, M. Eugênia; KATO, Mary A. Null subjects in European and Brazilian Portuguese. Journal of Portuguese Linguistic, 4: 11-52, 2005.

CARDINALETTI, Anna. Toward a cartography of subject positions. In: Rizzi, Luigi (ed.), The Structure of CP and IP. The Cartography of Syntactic Structures, Volume 2, New York, Oxford University Press, 115-165, 2004.

CARDINALETTI, Anna. Cross-linguistic variation in the syntax of subjects. Linguistic Variation in the Minimalist Framework, Oxford, Oxford University Press, pp. 82-107, 2014.

CARDINALETTI, Anna. STARKE, Michal. The typology of structural deficiency: a case of the three classes of pronouns. In: van RIEMSDIJK, H. (ed.). Clitics in the language of Europe. Berlin: Mouton et Gruyter, 1999. p. 41-109.

COSTA, João. Word order and discourse-configurationality in European Portuguese. In: COSTA, J. (ed.) Portuguese syntax: New comparative studies. Oxford: Oxford University Press, 2000. 
COSTA, João. Spec, IP ou Deslocado? Prós e contras das duas análises dos sujeitos préverbais. DELTA, 17(1), p. 283-304, 2001.

COSTA, João. PB e PE: orientação para o discurso importa?. Estudos da Língua(gem) Estados diacrônicos e sincrônicos da Língua Portuguesa.Vitória da Conquista, v. 8, n. 1, 123143, 2010.

COSTA, João; DUARTE, Inês. Preverbal subjects in null subject languages are not necessarily dislocated. Journal of Portuguese Linguistics, 1, 159-175, 2002.

COSTA, João; DUARTE, Inês; SILVA, Claudia. Construções de redobro em português brasileiro: sujeitos tópicos vs. Soletração do traço de pessoa. Leitura, n. 33, p 135- 145, 2004.

CYRINO, Sonia M. L., DUARTE, M. Eugênia. L. e KATO, Mary A. Visible subjects and invisible clitics in Brazilian Portuguese. In: M. A. Kato \& E. V. Negrão (Orgs.). p. 55-104, 2000.

DUARTE, M. Eugênia. Do pronome nulo ao pronome pleno: A trajetória do sujeito no português do Brasil. In I. Roberts and M. A. Kato (eds.), Português Brasileiro: Uma viagem diacrônica. Campinas: Editora da UNICAMP, p. 107-128, 1993.

DUARTE, M. Eugênia. A perda do princípio "Evite pronome" no Português Brasileiro. Tese de Doutorado, Universidade Estadual de Campinas, 1995.

DUARTE, M. Eugênia. The loss of the "avoid pronoun" principle in Brazilian Portuguese. In M. A. Kato and E. Negrão (eds.), The Null Subject Parameter in Brazilian Portuguese. Frankfurt \& Madrid: Vervuert-Iberoamericana, p. 17-36, 2000.

DUARTE, M. Eugênia; SOARES DA SILVA, Humberto. Microparametric variation in Spanish and Portuguese: The null subject parameter and the role of the verb inflectional paradigm. In: KATO, M; ORDONEZ, Francisco (Orgs). The Morphosyntax of Portuguese and Spanish in Latin America. Oxford University Press, p. 1-26, 2016.

DUARTE, Inês; FIGUEIREDO SILVA, Maria Cristina. The Null Subject Parameter and the Structure of the Sentence in European and Brazilian Portuguese. In: WETZELS, L.; COSTA, J.; MENUZZI, S. (eds.), The Handbook of Portuguese Linguistics, Wiley Blackwell, 234-253, 2016.

FIGUEIREDO SILVA, Maria Cristina. A posição do sujeito no português brasileiro: frases finitas e infinitivas. Campinas: Editora da Unicamp, 1996.

FIGUEIREDO SILVA, Maria Cristina. Main and embedded null subjects in Brazilian Portuguese. In: Kato \& Negrão (Eds), The Null Subject Parameter in Brazilian Portuguese. Frankfurt \& Madrid: Vervuert-Iberoamericana, p. 127-146, 2000.

GALVES, Charlotte. A sintaxe do português brasileiro. Ensaios de Linguística, 13: 31-49, 1987.

GALVES, Charlotte. Tópicos, sujeitos, pronomes e concordância no português brasileiro. Cadernos de Estudos Linguísticos, 34: 7-21, 1998. 
GALVES, Charlotte. Ensaios sobre as gramáticas do português. Campinas: Editora da Unicamp, 2001.

KATO, Mary A. Tópicos como predicados alçados. Cadernos de Estudos Linguísticos, 34: 67-76, 1998.

KATO, Mary A. Strong pronouns, weak pronominals and the null subject parameter. Probus 11: 1-37, 1999.

KATO, Mary A. Comparando o Português da América com o Português de Portugal e com outras línguas. Museu da Língua Portuguesa. Disponível em: http://www.museudalinguaportuguesa.org.br/colunas_interna.php?id_coluna=13, 2006.

KATO, Mary A. Expletivos nulos e construções de tópico/sujeito no português brasileiro. Cadernos de Estudos Linguísticos (57.1), p. 7-21, 2015.

KATO, Mary; DUARTE, M. Eugênia. Mudança paramétrica e orientação para o discurso. Comunicação apresentada no XXIV Encontro da Associação Portuguesa de Linguística, Universidade do Minho, Braga, 2008.

KATO, Mary; DUARTE, M. Eugênia. Restrições na distribuição de sujeitos nulos no Português Brasileiro. Veredas 18(1): 1-22, 2014.

LI, C. N.; THOMPSON, S. A. Subject and topic: a new typology of language. In: LI, C. N. (ed.). Subject and topic. New York: Academic Press Inc., 1976.

PILATI, E. S., NAVES, R. R. Desenvolvendo a hipótese da cisão da categoria pronominal no português brasileiro. In: Denilda Moura; Marcelo A. Sibaldo. Estudos e Pesquisas em Teoria da Gramática.1 ed.Maceió : EDUFAL, 2013, p. 233-253.

POLETTO, Cecília. The Higher Functional Field in the Northern Italian Dialects. Oxford: Oxford University Press, 2000.

PONTES, Eunice. O tópico no português brasileiro. Campinas, 1987.

QUAREZEMIN, Sandra. Alternância sintática no Português Brasileiro: sujeito ou tópico?. Comunicação apresentada no Anagrama (Grammatical Analysis and Corpora). Universidade de Lisboa, novembro de 2016.

QUAREZEMIN, Sandra. A arquitetura da sentença no Português Brasileiro: considerações sobre Sujeito e Tópico. Revista Letras, Curitiba, UFPR, n. 96, 196-218, 2017a.

QUAREZEMIN, Sandra. Ensinar Linguística na escola: um confronto com a realidade. Working Papers em Linguística, 18(2): 69-92, 2017 b.

QUAREZEMIN, Sandra. Construções com alternância sintática no Português Brasileiro. In: Antonelli, Roberto; Videsott, Paul; Glessgen, Martin (Orgs). Atti del XXVIII Congresso Internazionale di Linguistica e Filologia Romanza, BiLiRo 15, p. 594-606, 2018.

QUAREZEMIN, Sandra. A cartografia das posições de sujeito nas sentenças com redobro em 
português brasileiro. Comunicação apresentada no Encontro Nacional do Grupo de Trabalho de Teoria da Gramática da ANPOLL. Florianópolis, 28 e 29 de junho de 2018.

QUAREZEMIN, Sandra; CARDINALETTI, Anna. Non-topicalized preverbal subjects in Brazilian Portuguese, compared to Italian. Rivista Annali di Ca' Foscari. Serie occidentale, vol 51, 383-409, 2017.

RIZZI, Luigi. Null objects in Italian and the theory of pro. Linguistic Inquiry, 17: 501-557, 1986a.

RIZZI, Luigi. On the status of sublect clitics in Romance. In O. Jaeggli and C. Silva-Corvalán (eds.), Studies in Romance Linguistics. Dordrecht: Foris, p. 391-419, 1986 b.

RIZZI, Luigi. Relativized Minimality. Cambridge, Mass: The MIT Press, 1990.

RIZZI, Luigi. Early null subjects and root null subjects. Geneva Generative Papers, 1992.

RIZZI, Luigi. The Fine Structure of the Left Periphery. L. Haegeman (ed.) Elements of Grammar. Dordrecht: Kluwer, 281-337, 1997.

RIZZI, Luigi. On the position of Int(errogative) in the Left Periphery of the Clause, in: CINQUE, G; SALVI, G. (Eds.), Current Studies in Italian Syntax, Elsevier, Amsterdam, p. 287-296, 2001.

RIZZI, Luigi. On some properties of subjects and topics. In: BRUGÉ, L. et al (eds.). Proceedings of the XXX Incontro di Grammatica Generativa. Venezia, Cafoscarina, p. 203224, 2005.

RIZZI, Luigi. On the form of chains: Criterial positions and ECP effects. In $O n W h$ Movement, Lisa Cheng \& Norbert Corver (eds). Cambridge MA: The MIT Press, p. 97-133, 2006.

RIZZI, Luigi. On some properties of criterial freezing. In The Complementizer Phase: Subjects and Operators, E. Phoevos Panagiotidis (ed.). Oxford: OUP, p. 17-32, 2010.

RIZZI, Luigi. Focus, Topic and the cartography of the left periphery. In The Bloomsbury Companion to Syntax, Cristina Parodi \& Silvia Luraghi (eds), London: Bloomsbury, p. 43645, 2013.

RIZZI, Luigi. Some consequences of criterial freezing: Asymmetries, anti- adjacency and extraction from cleft sentences. In Functional Structure from Top to Toe. The Cartography of Syntactic Structures, Vol. 9, Peter Svenionius (ed.), Oxford: OUP, p. 19-45, 2014.

RIZZI, Luigi. Cartography, criteria, and labeling. In Beyond Functional Sequence, Ur. Shlonsky (ed.), Oxford: OUP, p. 314-338, 2015.

RIZZI, Luigi. Cartography and explanation. Minicurso ministrado no $3^{\text {rd }}$ International Meeting of Syntax, Semantics, Interfaces and Cognition (EISSI). Florianópolis, junho de 2018.

RIZZI , Luigi; SHLONSKY, Ur. Satisfying the subject criterion by a non Subject: English 
locative inversion and heavy NP shift. In Mara Frascarelli (ed.), Phases of interpretation, Berlin: Mouton de Gruyter, p. 341-361, 2006.

RIZZI , Luigi; SHLONSKY, Ur. Strategies of subject extraction. In Hans Martin Gärtner \& Uli Sauerland (eds.), Interfaces + recursion = Language? Chomsky's minimalism and the view from syntax-semantics, Berlin: Mouton de Gruyter, p. 115-160, 2007.

SILVA, Claudia Roberta Tavares. 2006. Duplicação de sujeitos pré-verbais no francês, no inglês e no português brasileiro: uma análise não-unificada. Revista de Estudos Linguísticos, Belo Horizonte, v. 14, n. 2, p. 185-210.

SHLONSKY, Ur; RIZZI , Luigi. Criterial freezing in small clauses and the cartography of copular constructions. In Proceedings Workshop Freezing: Theoretical Approaches and Empirical Domains, Susanne Winkler. Tübingen: Tübingen University, no prelo.

SOUZA, E. M. O uso do pronome 'eles' como recurso de indeterminação do sujeito. Dissertação (Mestrado em Estudos Linguísticos) - Faculdade de Letras da UFMG, Belo Horizonte, 2007.

Sandra Quarezemin $\underline{\text { sandra@cce.ufsc.br }}$

Recebido em: 28 fev. 2019

Aceito em: 21 Abril. 2019

Publicado em: Junho de 2019 